\title{
Increased mechanical robustness of piezoelectric magnetoelastic vibrational energy harvesters
}

Alcala-Jimenez, L.R.; Passer Jensen, Thomas; Lei, A.; Thomsen, E.V.

Published in:

Microelectronic Engineering

Link to article, DOI:

10.1016/j.mee.2018.12.003

Publication date:

2019

Document Version

Peer reviewed version

Link back to DTU Orbit

Citation $(A P A)$ :

Alcala-Jimenez, L. R., Passer Jensen, T., Lei, A., \& Thomsen, E. V. (2019). Increased mechanical robustness of piezoelectric magnetoelastic vibrational energy harvesters. Microelectronic Engineering, 207, 19-26.

https://doi.org/10.1016/j.mee.2018.12.003

\section{General rights}

Copyright and moral rights for the publications made accessible in the public portal are retained by the authors and/or other copyright owners and it is a condition of accessing publications that users recognise and abide by the legal requirements associated with these rights.

- Users may download and print one copy of any publication from the public portal for the purpose of private study or research.

- You may not further distribute the material or use it for any profit-making activity or commercial gain

- You may freely distribute the URL identifying the publication in the public portal 


\title{
Increased Mechanical Robustness of Piezoelectric Magnetoelastic Vibrational Energy Harvesters
}

\author{
L. R. Alcala-Jimenez *, T. Passer*, A. Lei*, and E. V. Thomsen* \\ ${ }^{*}$ Department of Micro and Nanotechnology, Technical University of Denmark, DK-2800 Kgs. Lyngby, Denmark
}

\begin{abstract}
This work presents a cantilever based broadband piezoelectric magnetoelastic vibration energy harvester with increased mechanical robustness. The energy harvester is fabricated using $\mathrm{KOH}$ etching to define the cantilever and the proof mass is made using micromachined Fe foils which together with a pair of miniature magnets provides the magnetoelastic properties. KOH etching leads to very sharp corners at the anchoring point of the cantilever which makes the cantilever fragile. The mechanical robustness of the energy harvesters is increased using a lithography-free two-step fabrication process where a thermal oxidation is used for corner rounding. The corner rounding at the anchoring point lowers the stress concentration and thereby increases the robustness of the device. The radius of curvature for the corner depends linearly on the thickness of the oxide. Both enhanced and non-enhanced beams are excited at increasing frame accelerations. The conventional beams break at frame accelerations of around $3 \mathrm{~g}$ while the enhanced break at almost twice as much, $5.7 \mathrm{~g}$. The devices are characterized electrically by impedance measurements in both their linear and non linear regime. The magnetoelastic behaviour can be adjusted by varying the beam-magnet distance which allows for both spring softening and spring hardening.
\end{abstract}

Keywords - MEMS, Energy harvester, Robust, Piezelectric, Magnetoelastic, Ambient vibration.

\section{INTRODUCTION}

The need to power wearable devices, wireless sensor systems, and devices for the Internet of Things (IOT) increases the demand on small-scale power sources. Power source requirements for such systems are physical dimensions in the millimeter-scale range and long lifetime. Conventional batteries imply continuous and sometimes expensive replacement cost. Furthermore, they impose a limit on system miniaturization. This means that a replacement for conventional batteries must be found. Miniaturized cantilever-based vibrational energy harvesters (VEHs) capable of transforming energy from the mechanical domain, i.e. vibrations, into energy in the electrical domain have recently been receiving increasing interest in this field of application. There are three main approaches to harvesting energy from vibrations, which involve the use of either electrostatic [1-3], electromagnetic [4], [5] or piezoelectric [6-10] principles. Among these three different types of VEHs, piezoelectric-based VEHs have attracted much attention because it is feasible to produce them in millimeterscale whilst having large power densities. In [11-13] a power density of $35.4 \mathrm{~mJ} / \mathrm{cm}^{3}$ is reported for a piezoelectric energy harvester and this value can be compared to results obtained for electrostatic and electromagnetic devices, where power densities of $4 \mathrm{~mJ} / \mathrm{cm}^{3}$ and $24.8 \mathrm{~mJ} / \mathrm{cm}^{3}$ [11] have been reported, respectively.

Most ambient vibrations are characterized by a low frequency range $(<200 \mathrm{~Hz})$ [14] and accelerations are typically below $1 \mathrm{~g}$. In order to tune in to the low frequencies needed, the common approach is to increase the cantilever length and reduce its thickness or increasing the weight of the proof mass often attached to the beam. This leads to very high aspect ratios and therefore to very fragile structures due to stress concentration [15-17] at the anchoring point. Another method for lowering the operation frequencies has been presented by [18], where a five-step fabrication process is performed. It consists of spray coating resist on the backside of the beam after defining the cantilever, then a lithography process is performed and finally deep holes, with a radius of $25 \mu \mathrm{m}$ and a depth of $45 \mu \mathrm{m}$, are etched on the Si hinge. The resulting structure presented a resonant frequency of $802 \mathrm{~Hz}$, this value can be compared to the regular cantilevers, i.e. where no holes were etched on the structure, for which a resonant frequency of $845 \mathrm{~Hz}$ was obtained. Regarding the harvested power, it was increased $18 \%$ compared to the standard structures.

Besides the low frequency range of ambient vibrations, most of them do not occur at a specific frequency, but over a wide spectrum of frequencies. Therefore, research has been carried out in the field of broadband energy harvesting, where an external force is typically used to provide a potential energy landscape suitable for broad-band energy harvesting. Depending on the sources of the forces two types of methods can be distinguished: active and passive methods. In the active method the device needs to be continuously either powered or manually modified. Therefore, unless the gained energy is larger than the energy consumed by the powering source, it is of no further interest to investigate this type. On the other hand, a passive method requires to be only intermittently powered or even not powered at all. Magnetoelastic VEHs use an external magnetic setup that interacts with the beam by implementing either a magnet or a ferromagnetic foil on the beam [19-22], as shown in Fig. 1. Adding this magnetic setup will modify the spring constant of the beam according to the distance between the magnets and the beam, $a$, and the distance between the magnets, $b$. The spring constant will either become lower or higher depending on whether a softening [23] or hardening [24] effect takes place, respectively. Another passive method is the use of beam stoppers [25] that interact with the beam. This interaction takes place when the beam engages with 


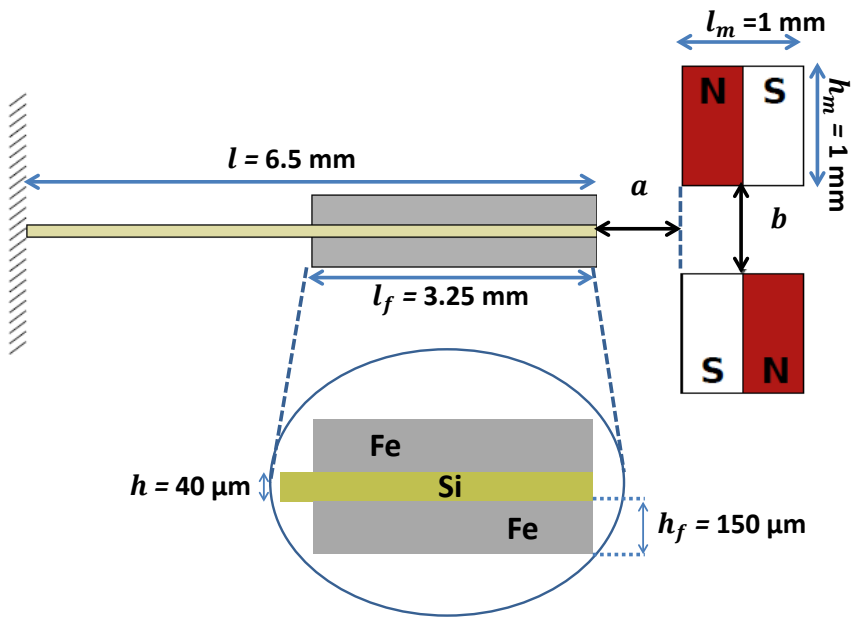

Fig. 1. Side view of the silicon based piezoelectric magnetoeleastic vibrational energy harvester system. The proof mass is realized by gluing pieces of $\mathrm{Fe}$ foil to the silicon cantilever.

the stopper and the effective spring constant is therefore changed. Furthermore, by applying stoppers to the energy harvesting system the beam bending is restricted to lower values, therefore they are less prone to fracture.

Typical VEHs use silicon wafers as substrates due to the well established Si micro- and nanofabrication techniques, nevertheless, studies have also been performed using metal substrates instead [26], [27]. This is due to their higher plastic deformation which, compared to the highly brittle material that $\mathrm{Si}$ is, clearly displays an advantage in the relative displacement that the beams can undergo. These larger deflections translate to larger strains in the piezoelectric material and, therefore, higher output power. Another advantage of these metal-based structures is that a lower resonant frequency can be achieved even without the use of a seismic mass [26]. However, an important drawback of using metal substrates is the restrictions in micro- and nano-processing techniques that these metals impose.

VEHs based on silicon beam structures are often defined using $\mathrm{KOH}$ etching as a simple means of fabrication as the etch stops on (111) silicon planes. $\mathrm{KOH}$ etched structures have very sharp corners at the intersection of the (111) and (100) planes and the radius of curvature is very small and values of $0.01 \mu \mathrm{m}$ have been reported by [28] for structures etched in KOH. Such structures are fragile due to stress concentration at the sharp corner and this has been used to fabricate micro structures that are easily removed [28]. For sensors and actuators this fragility is, of course, of concern and in pressure sensor applications the sharp corners can be rounded by an etching process to increase the fracture strength [29-31]. When designing beams for VEHs it is important to know the radius of curvature of the corner. Once this is known, the theory of stress concentration factors [15-17] can be used to estimate the maximum stress at the clamping point, or the structure can be simulated by finite element modelling using the radius of curvature to describe the structure at the clamping point. For $\mathrm{KOH}$ etched structures the stress concentration factors reported in [28] show that the maximum stress is reduced significantly when the radius of curvature is increased and the stress concentration factor decreases from around 15 for structures having a radius of curvature of $0.01 \mu \mathrm{m}$ to values around 1.5 when the radius of curvature is larger than $3 \mu \mathrm{m}$. As corner rounding has a significant effect on the stress concentration it is important to be able to predict the radius of curvature at the clamping point.

This study presents a fabrication process for a mechanically robust piezo electric cantilever-based VEH suitable for magnetoelastic energy harvesting. The cantilever is defined in silicon using $\mathrm{KOH}$ etching and the increased mechanical robustness is obtained by rounding the sharp $\mathrm{KOH}$ etched corner at the anchoring point of the beam. This corner rounding is performed by oxidation using a two-step batch fabrication process that increases the robustness of the beam without compromising beam dimensions. During oxidation the corner is rounded due to two dimensional diffusion of the oxidant and the radius of curvature can be predicted accurately. An AlN thin film is deposited on the beam providing the piezo electric properties and $\mathrm{Fe}$ foils are used both as proof mass and to provide magnetoeleastic behaviour.

The article is organized as follows: First, the materials and methods are described and the fabrication process used to fabricate the VEHs is presented. Secondly, the measurement setups for both characterizing the mechanical robustness of the devices and their piezo magnetoelastic behaviour is explained. Then, results and discussions are presented and finally, the article ends with the conclusions.

\section{MATERIALS AND METHODS}

\subsection{Fabrication of energy harvesters}

The main steps of the fabrication process are shown in Fig. 2 and the dimensions of the device are given in Table I. The process begins with a (100) oriented $\mathrm{Si}$ substrate having a resistivity of $0.0015 \Omega \mathrm{cm}$, Fig. 2a, which allows the silicon substrate to be used as a bottom electrode for the piezo electric material. The etch mask for $\mathrm{KOH}$ etching is a $3000 \mathrm{~nm}$ thick wet oxide layer which is grown on both sides of the wafer. Then a lithography process is performed followed by a BHF etching of the oxide layer to define the etch mask, Fig. 2b. After this, the beam is made using $\mathrm{KOH}$ etching, Fig. 2c, and the oxide layer is removed by etching in BHF, Fig. 2d. Then, the corner rounding step takes place, which consists of growing a $1000 \mathrm{~nm}$ wet oxide layer at $1100^{\circ} \mathrm{C}$ and right afterwards stripping it, as shown in Fig. 2e-f, respectively. After this, the piezo electric material and top and bottom electrodes are deposited by evaporation and sputtering of Pt/AlN/Pt/Ti with thicknesses of $50 \mathrm{~nm} / 400 \mathrm{~nm} / 120 \mathrm{~nm}$ $/ 10 \mathrm{~nm}$, respectively, Fig. 2g. A lithography process is then performed on that side of the wafer, Fig. 2h, with an opening for the top electrode definition using AZ 4562 resist with a thickness of $10 \mu \mathrm{m}$. The three-stack layer is etched in Aqua Regia at $80^{\circ} \mathrm{C}$, Fig. $2 \mathrm{i}$, with the concentrations shown in Table II. However, the lithography and etching process is 
TABLE I

DIMENSIONAL AND MATERIAL PARAMETERS FOR THE ENERGY HARVESTING SYSTEM.

\begin{tabular}{ll}
\hline \hline Device parameter & Value \\
\hline \hline Beam length $(l)$ & $6.5 \mathrm{~mm}$ \\
Beam thickness $(h)$ & $40 \mu \mathrm{m}$ \\
Beam width & $6 \mathrm{~mm}$ \\
Foil length $\left(l_{f}\right)$ & $3.25 \mathrm{~mm}$ \\
Foil thickness $\left(h_{f}\right)$ & $100 \mu \mathrm{m}$ \\
Foil width & $6 \mathrm{~mm}$ \\
Magnet length $\left(l_{m}\right)$ & $1 \mathrm{~mm}$ \\
Magnet thickness $\left(h_{m}\right)$ & $1 \mathrm{~mm}$ \\
Magnet width & $5.5 \mathrm{~mm}$ \\
Magnetization & $750 \mathrm{~A} / \mathrm{m}$ \\
Si Young modulus & $150 \mathrm{GPa}$ \\
\hline \hline
\end{tabular}

TABLE II

AQUA REGIA COMPOSITION AND APPROXIMATE ETCH RATES.

\begin{tabular}{ll}
\hline \hline Parameter & Value \\
\hline \hline $\mathrm{H}_{2} \mathrm{O}$ & $400 \mathrm{ml}$ \\
$\mathrm{HCl}$ & $350 \mathrm{ml}$ \\
$\mathrm{HNO}_{3}$ & $50 \mathrm{ml}$ \\
Etch rate in $\mathrm{Pt}$ & $\approx 10 \mathrm{~nm} / \mathrm{min}$ \\
Etch rate in $\mathrm{AlN}$ & $\approx 26 \mathrm{~nm} / \mathrm{min}$ \\
\hline \hline
\end{tabular}

performed three times due to the high temperatures that the resist must withstand during the Aqua Regia etchings. Once this is completed, another layer of AZ 4562 resist is spun on the same side of the wafer, Fig. $2 \mathrm{j}$, this time with a thickness of $5 \mu \mathrm{m}$. This serves as masking material for the release of the beams, which is performed by using an STS ICP Advanced Silicon Etcher system, Fig. 2k. After stripping the resist the devices are then ready to be diced out, this is done using a Disco Automatic dicing saw, model DAD321. The normal procedure before dicing is to use electrostatic tape that holds the wafer still while dicing and once it is done this tape is just pealed off. However, due to the fragility of the beams the tape must be pealed off while the wafers are immersed in acetone.

The final step is to attach Fe foils to the beam, Fig. 2l. These foils act both as proof mass, lowering the resonant frequency, and as an integrated part of the magnetoelastic harvesting scheme. It is important that the ferromagnetic foil that is attached to both sides of the beam is as flat as possible, without bending parts, therefore guaranteeing a structurally symmetric foil. The foil is milled in a computer numeric control milling machine from Minitech Machinery Corporation. A methyl methacrylate (PMMA) surface is normally used to hold the substrate while the milling takes place. In order to hold still the foil on the PMMA surface, electrostatic tape is used, as seen in Figure 3, where the blue spots are the residues of the tape once the milling has been completed and the ferromagnetic foil removed. For removing the foil from the electrostatic tape the whole substrate is submerged into an acetone bath. After this step, the foil is incorporated to the beam by a pick-andplace operation, i.e. it is glued on both sides of the tip of the beam, Fig. 21.

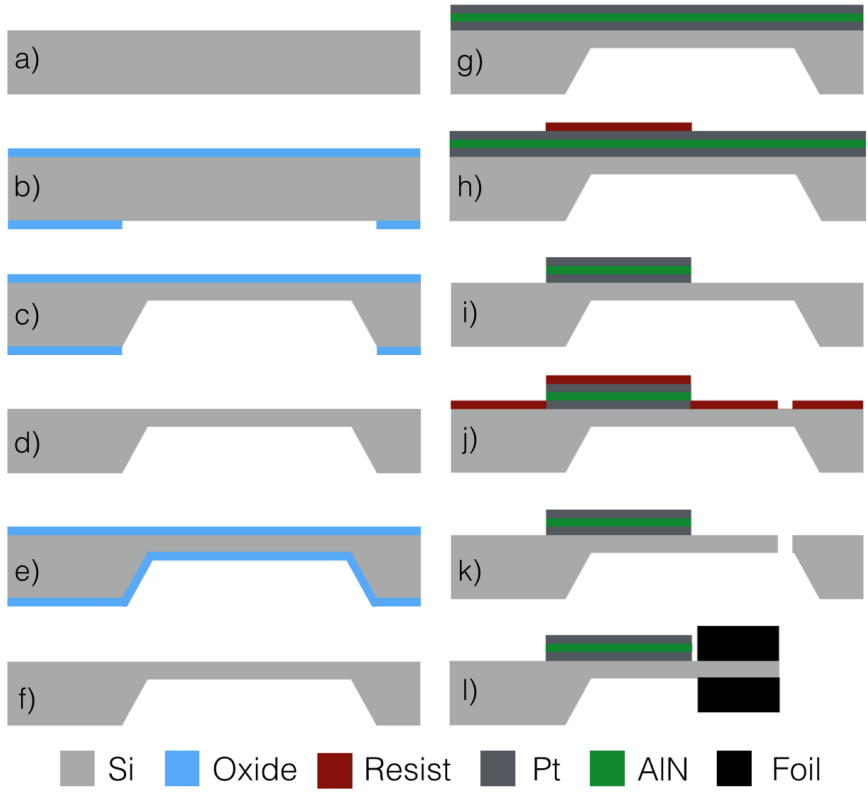

Fig. 2. Main steps of the process flow followed to fabricate the VEHs. a) $\mathrm{Si}$ wafer. b) Thermal growth of an oxide layer to serve as masking material. c) $\mathrm{KOH}$ etching. d) $\mathrm{BHF}$ etch to remove oxide. e) Oxide growth to round corners. f) BHF etch of oxide layer. g) Three-stack deposition of top and bottom electrodes and piezoelectric AlN. h) Lithography process to mask the etching of the three layered stack. i) Aqua regia etch of the three layered stack. j) Lithography process for the beam release. k) Beam release by dry etching. 1) Finally, devices are diced and ferromagnetic foil is glued on both sides of the beam.

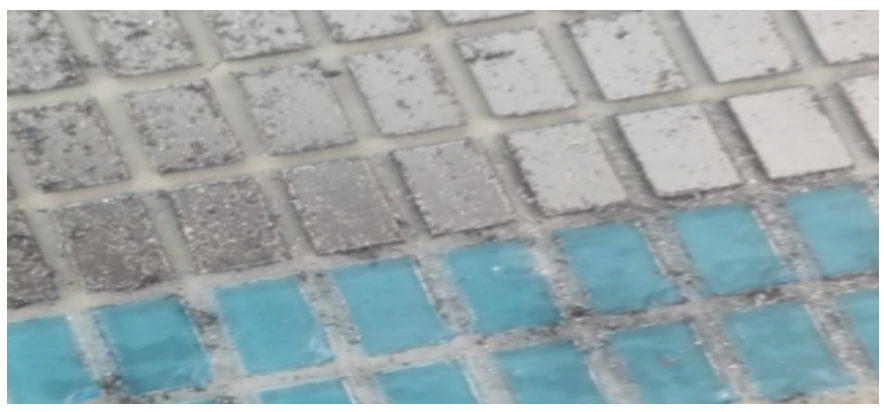

Fig. 3. Ferromagnetic foil after the milling process. The foil is fixed to a PMMA substrate (white) using electrostatic tape (blue) which allows the foil to be diced in a controlled way.

\subsection{Measurement setups for characterization}

The characterization of the devices consists of two parts. In the first part, the response of the beams in the mechanical domain is analyzed, i.e. the piezoelectric and electrode layers are not implemented in the beams, only the ferromagnetic foil is included to act as proof mass. The purpose of this part of the analysis is to investigate the mechanical robustness of the devices. In the second part, the piezoelectric and electrode layers are included on the beams, and the devices are characterized in terms of impedance, i.e. their performance in the electrical domain is analyzed and the magnetoelastic properties of the VEHs are determined. 


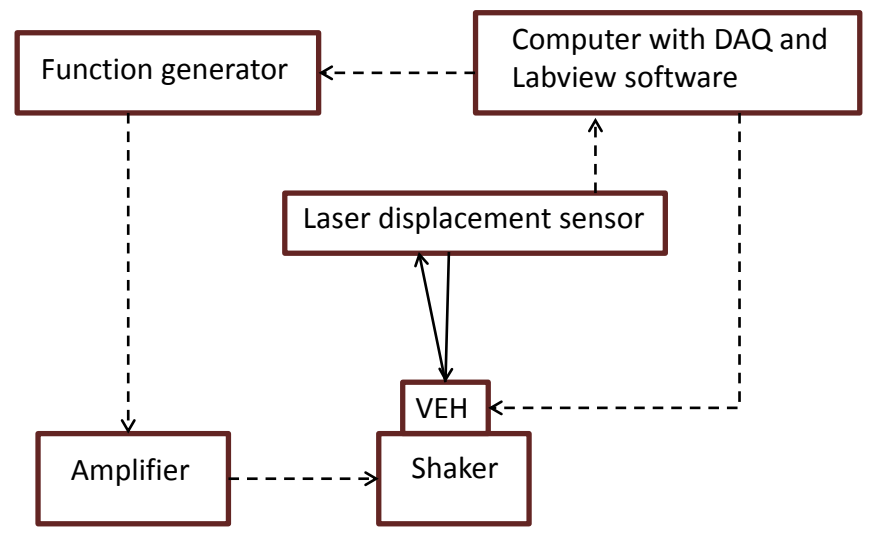

Fig. 4. Schematic of the setup used for characterizing the mechanical properties of the energy harvesters.

2.2.1 Mechanical domain: To characterize the beams in mechanical terms the setup shown schematically in Fig. 4 is used. A sinusoidal signal is generated from an Agilent $33220 \mathrm{~A}$ waveform generator and amplified by a Pioneer VSX405RDS MKII amplifier, which is directly connected to a B\&K Minishaker 4810, which acts as the excitation source for the devices. During vibrational testing the devices are mounted to a sample holder which is screwed to the table of the shaker. The deflection of the beams as function of time is measured by a Micro-Epsilon ILD2300-10 laser triangulation displacement sensor, which is placed above the sample holder. The acceleration of the sample holder for a given harmonic driving signal was previously determined from a deflectiontime measurement.

The devices tested are both the enhanced ones, i.e. those that underwent a corner rounding treatment, and the regular beams having a sharp corner at the beam anchoring point. The specific dimensions are the same as the ones listed in Table I. The resonant frequency of the devices was found by sweeping the frequency and measuring the deflection of the beam.

To investigate the mechanical robustness of the devices they were excited at different levels of acceleration and frequency sweeps were performed. The acceleration of the sample holder was increased by changing the magnitude of the signal driving the shaker until the beam under investigation broke. The frequency sweep was performed with a step size of $0.01 \mathrm{~Hz}$ to avoid breaking the beams due to a too large step in frequency. In order to perform a statistical analysis of the results, the Weibull method [32] was used.

2.2.2 Electrical domain: The electrical characterization of the fabricated devices was performed using impedance measurements. For this purpose an Agilent E4990A Impedance Analyzer was used. The analyzer was connected to an Agilent 4294A impedance probe, Fig. 6, which was connected to the device under investigation. The impedance measurements were performed using a signal amplitude of $50 \mathrm{mV}$. The top electrode of the beam is in contact with a pogo-pin from the sample holder and a set of other pogo-pins secured it by

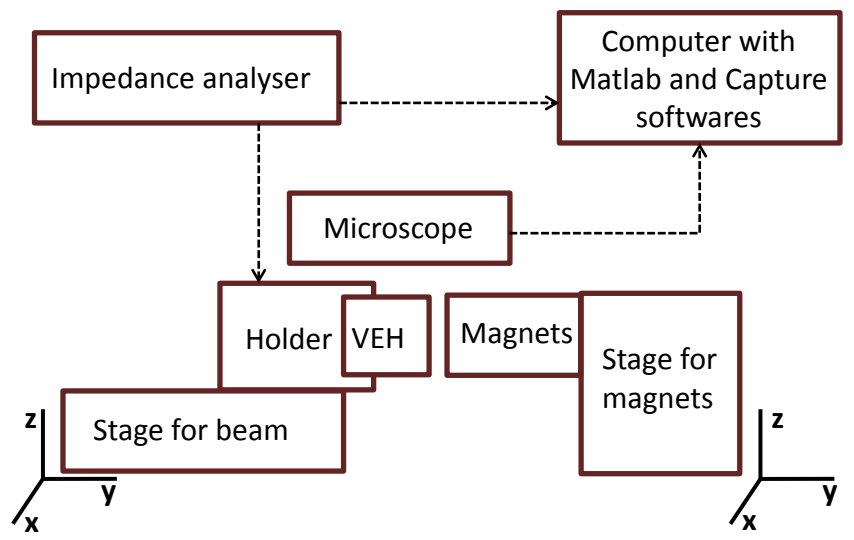

Fig. 5. Schematic of the setup used for characterizing the electrical properties of the devices.

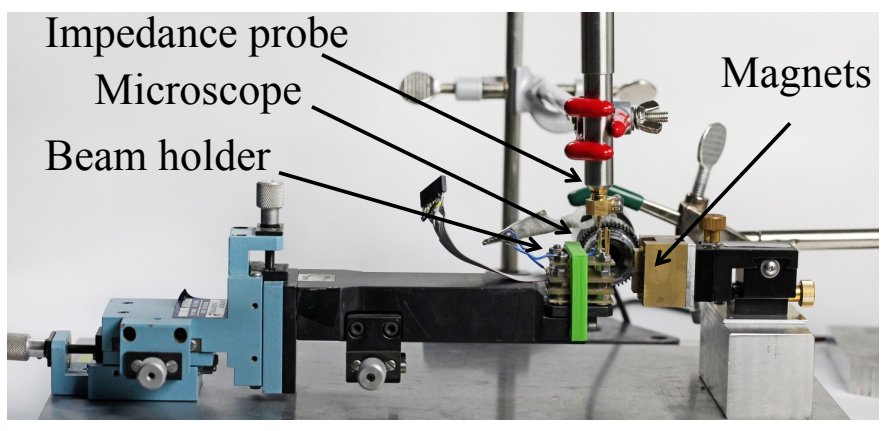

Fig. 6. Setup used for the electrical characterization.

pressing on the frame of the silicon chip, Fig. 7a. Part of the backside frame structure of the device lies on a metal structure which is connected to the impedance probe. Furthermore, to analyze the non-linear response of the system, a pair of $\mathrm{NdFeB}$ magnets are included into the system, Fig. $7 \mathrm{~b}$. In order to have a precise control over the positioning of the magnets in the three spatial dimensions, a XYZ 300 TR stage model from Quater Research and Development is used, Fig. 6.

When using the magnetic setup, the distance between the magnets and the beam, $a$, was measured by a Dino-Lite edge digital microscope model AZ7915MZTL together with
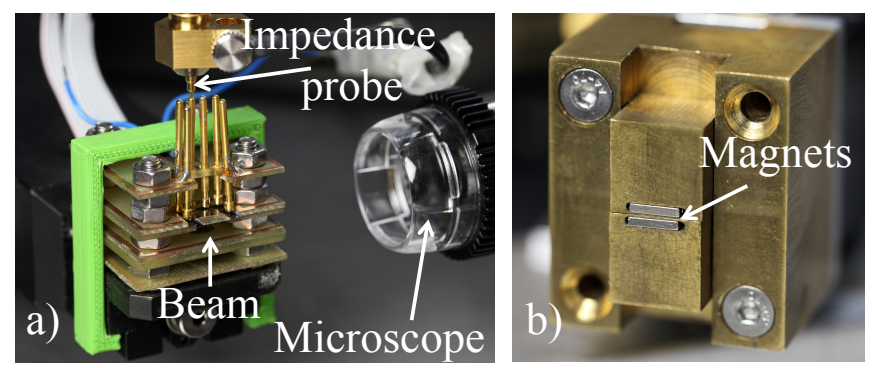

Fig. 7. Part of the setup used for the electrical characterization. a) Beam holder with the pogo-pins holding the beam still and one of them connected to the impedance setup. Also, shown is the microscope used for measuring distances. b) Holder for the magnets with the rectangular magnets located at the center of the fixture. 
DinoCapture 2.0 software [33]. For all measurements the distance between the magnets was $b=520 \mu \mathrm{m}$.

The impedance measurements were used to determine the resonant frequency of the beam, the effective spring constant, and the capacitance of the piezo electric material.

The effective spring constant of the beam, $k_{\text {eff }}$, was calculated from the resonant frequency of the beam, $f_{r}$, determined from the impedance measurements. The spring constant was found using $k_{\text {eff }}=\left(2 \pi f_{r}\right)^{2} m_{\text {eff }}$, where the value of the effective mass, $m_{\text {eff }}$, was found as described in [34]. The capacitance of the piezo electric layer, $C$, was calculated from an impedance measurement outside the resonant region of the impedance spectrum using $C=1 /(2 \pi f \cdot|Z|)$ where $f$ is the frequency and $|Z|$ is the magnitude of the impedance. The permittivity of the piezo electric layer, $\epsilon_{r}$, was found from the capacitance using $C=\epsilon_{r} \epsilon_{0} d / A$, where $d$ is the piezoelectric film thickness, $A$ the area of the electrodes, and $\epsilon_{0}$ is the vacuum permittivity.

\section{RESULTS AND DISCUSSION}

\subsection{Corner rounding}

The fabrication process for the devices where corner rounding was implemented, was designed using the Silvaco ATHENA [35] process simulator. The simulation takes into account the two dimensional diffusion of the oxidant and the different oxide growth rates on the (111) and (100) silicon surfaces due to the substrate orientation dependency of the linear rate constant. The difference in growth rates is, however, quite small for the structures investigated here. Furthermore, the simulation takes into account that the linear rate constant is increased due to the high phosphorous doping level of the substrate. The simulation code is included in the appendix. Fig. 8 shows the results from the simulation. Fig. 8 a shows the corner of the beam after the $\mathrm{KOH}$ etch which is implemented as a geometrical etch in the simulation code. Fig. $8 \mathrm{~b}$ shows the beam after 40 minutes of oxidation at $1100{ }^{\circ} \mathrm{C}$. At this point the oxide thickness is $619 \mathrm{~nm}$ and $624 \mathrm{~nm}$ on the (100) and (111) surfaces, respectively. Fig. 8c shows the corner structure after 80 minutes of oxidation and the oxide thicknesses are $876 \mathrm{~nm}$ and $881 \mathrm{~nm}$ on the corresponding (100) and (111) surfaces. The total oxidation time is 126 minutes and the final oxide thicknesses on the (100) and (111) surfaces are $1100 \mathrm{~nm}$ and $1105 \mathrm{~nm}$, respectively. As expected, the difference in oxide thickness on the (100) and (111) surfaces is very small. Finally, Fig. 8d shows the corner structure after the oxide layer has been removed.

Fig. 9 shows SEM images of the corner structure of the beam just after the $\mathrm{KOH}$ etch, Fig. 9a, and after the corner rounding has been performed, Fig. 9b. The effectiveness of the corner rounding process is clearly seen as an increased radius of curvature. The radius of curvature after the corner rounding process is $1.4 \mu \mathrm{m}$.

Fig. 9b compares the SEM image with the result from the simulation as presented on Fig. 8d. The simulation result is shown as the light structure on top of the SEM image. The solid red line shows the location of the surface of the
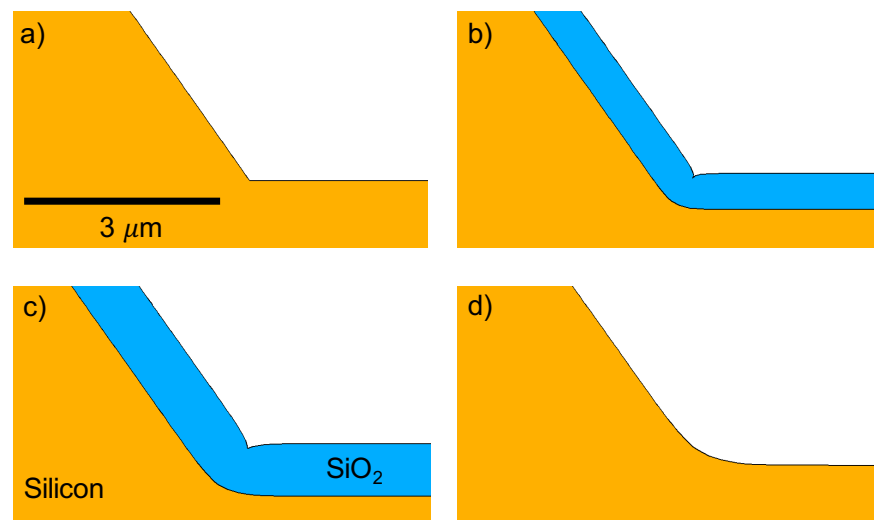

Fig. 8. Results from the corner rounding process simulation. a) As etched, b) After 40 minutes of oxidation, c) After 80 minutes of oxidation, d) After 126 min of oxidation and removal of the oxide.
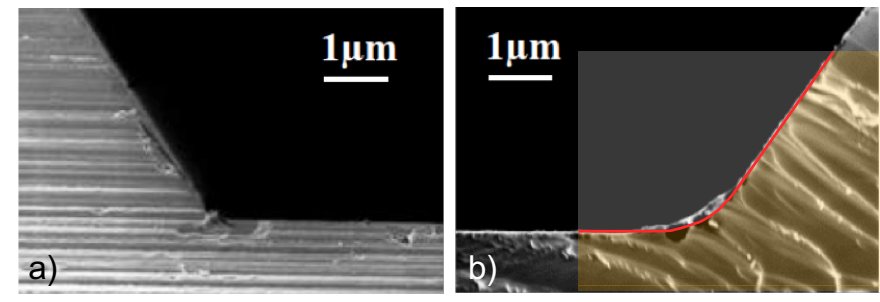

Fig. 9. SEM images of the corner structure of the beam a) before and b) after the oxide growth. The simulation result, Fig. $8 \mathrm{~d}$, is shown as the transparent structure overlaid on the SEM image. The solid red line shows the location of the surface of the simulation structure.

simulation structure. The shape calculated by the process simulation tool coincides with the shape obtained from the SEM investigation. Therefore, the simulation tool can be used to design a suitable corner rounded structure.

To further investigate the corner rounding process, simulations were carried out using both highly doped (having a phosphorous concentration of $1.0 \times 10^{20} \mathrm{~cm}^{-3}$ ) and undoped substrates. The simulations were performed at oxidation temperatures of $1000{ }^{\circ} \mathrm{C}, 1050{ }^{\circ} \mathrm{C}$ and $1100{ }^{\circ} \mathrm{C}$, respectively. The oxidation time was varied from 10 minutes to 10 hours and the thickness of the oxide grown on the (100) silicon surface was extracted from the simulation structure. For each simulation the shape of the corner was extracted and a circle was fitted to the datapoints allowing determination of the radius of curvature. The results are shown on Fig. 10. The radius of curvature is observed to depend linearly on the oxide thickness for the range of oxide thicknesses investigated. The solid line is a fit to all datapoints and the radius of curvature, $r$, depends on the oxide thickness $t_{\mathrm{ox}}$ as

$$
r=\alpha+\beta t_{\mathrm{ox}}
$$

where $\alpha=153 \pm 17 \mathrm{~nm}$ and $\beta=1.11 \pm 0.01$. This expression can be used to find a suitable oxide thickness to obtain a given radius of curvature. The slope of the line is close to 1 meaning that the radius of curvature is almost identical to the thickness of the oxide. As seen above, the difference in 


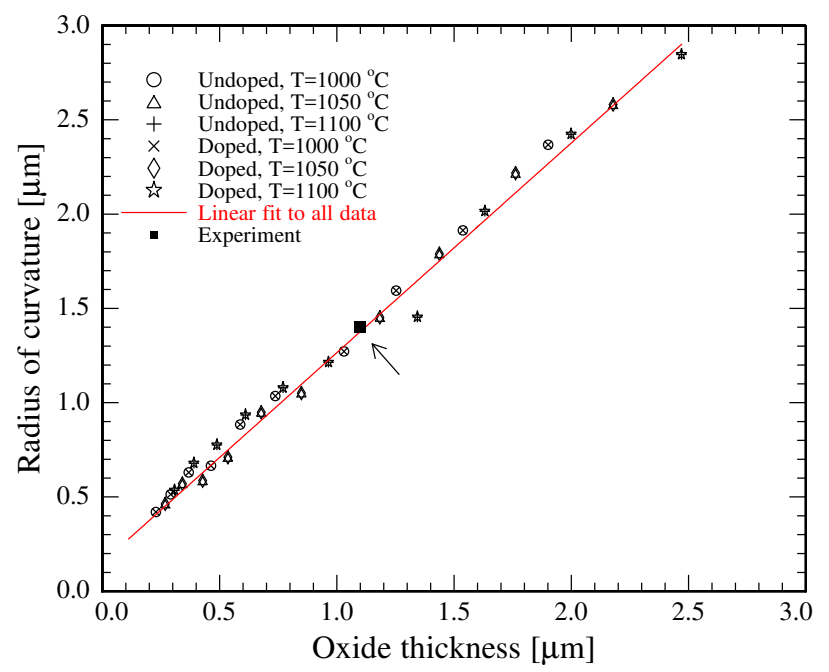

Fig. 10. Plot of the radius of curvature as function of the thickness of the oxide grown on the (100) silicon surface. The arrow points towards the solid square that represents the radius of curvature obtained experimentally, i.e. from the SEM image shown in Fig. 9b. The solid line is a linear fit to all datapoints.

TABLE III

FAILURE BASE ACCELERATIONS FOR BOTH THE REGULAR AND THE ENHANCED DEVICES.

\begin{tabular}{cccc}
\hline \hline \multicolumn{2}{c}{ Enhanced } & \multicolumn{2}{c}{ Regular } \\
\hline \hline$f_{r}[\mathrm{~Hz}]$ & acc. $[\mathrm{g}]$ & $f_{r}[\mathrm{~Hz}]$ & acc. $[\mathrm{g}]$ \\
\hline \hline 269.6 & 3.3 & 302.4 & 2.4 \\
283.0 & 2.4 & 284.5 & 3.5 \\
291.9 & 5.1 & 302.6 & 2.0 \\
276.7 & 7.4 & 306.2 & 3.1 \\
264.4 & 6.1 & 299.4 & 3.1 \\
268.6 & 7.0 & 296.9 & 3.8 \\
285.8 & 5.5 & 310.9 & 3.5 \\
281.3 & 8.3 & 335.5 & 2.9 \\
\hline $277.7 \pm 9.5$ & \multicolumn{3}{c}{$304.8 \pm 14.6$} \\
\hline \hline
\end{tabular}

thickness of the oxide grown on the (100) and (111) silicon surfaces is very small, on the order of a few nanometers. Therefore, the corner rounding effect occurs predominantly due to the two-dimensional diffusion of the oxidant at the corner structure. The radius of curvature determined from the SEM image shown in Fig. 9b is displayed as a black square and the experimental value is in very good agreement with the simulations and the linear model.

\subsection{Failure analysis}

Table III shows the measured resonant frequencies for enhanced and regular devices and the corresponding base acceleration where the devices failed due to breakage. In all cases, it was observed that the reason for the failure of the devices was that the beam broke at the point where it is attached to the silicon support structure, i.e. at the sharp corner defined by the $\mathrm{KOH}$ etch.

The cumulative failure distribution for the regular and enhanced devices, based on the data in Table III, are shown in
TABLE IV

PARAMETERS FOR THE FITTED WEIBULL DISTRIBUTION FUNCTIONS TO THE FAILURE ACCELERATIONS FOR THE REGULAR AND ENHANCED DEVICES.

\begin{tabular}{lll}
\hline \hline Parameter & Regular & Enhanced \\
\hline \hline Shape parameter & 6.73 & 3.49 \\
Scale parameter & 3.26 & 6.29 \\
Mean value [g] & 3.0 & 5.7 \\
Standard deviation $[\mathrm{g}]$ & 0.5 & 1.8 \\
\hline \hline
\end{tabular}

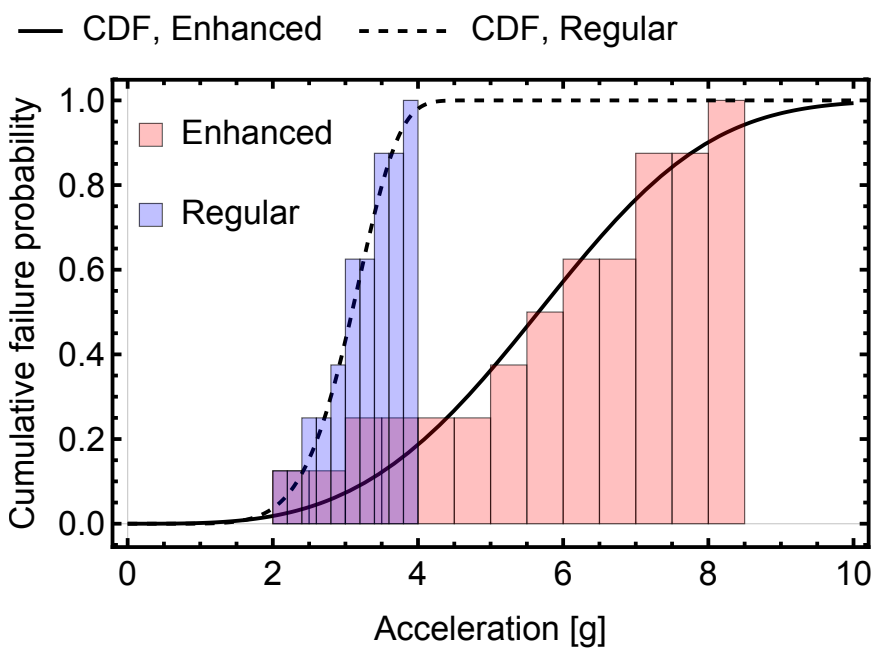

Fig. 11. Cumulative failure distribution for the regular and enhanced devices as function of base acceleration. The solid and dashed lines represents the fitted Weibull cumulative distribution functions (CDF) for the enhanced and regular devices, respectively.

Fig. 11 together with the fitted Weibull cumulative distribution functions. The characteristics of the fitted Weibull functions are listed in Tab. IV. For the regular devices the shape parameter (Weibull modulus) is $k_{r}=6.73$ and the scale parameter is $\lambda_{r}=3.26$. For the enhanced devices the corresponding numbers are $k_{e}=3.49$ and $\lambda_{e}=6.29$, respectively. As both shape parameters are larger than one, the rate of failure increases with acceleration.

Fig. 12 shows a plot of the failure accelerations as function of beam resonant frequencies for the regular and enhanced devices. The mean value of the failure acceleration is $3.0 \mathrm{~g}$ and $5.7 \mathrm{~g}$ for the regular and enhanced devices, respectively. The mean values are indicated by the dashed lines and the dotted lines correspond to one standard deviation. The data demonstrate that the corner rounding process increases the mechanical robustness of the energy harvesters.

The results found here are in good agreement with the findings of [29-31] that studied the use of wet etching or plasma etching induced corner rounding to increase the burst pressure of piezo resistive pressure sensors where $\mathrm{KOH}$ etching was used for defining the diaphragm. In [29], [30] the burst pressure of piezo resistive pressure sensors was increased from around $100 \mathrm{psi}$ for a device without corner rounding to $500 \mathrm{psi}$ when wet etching or plasma etching was used to increase the 


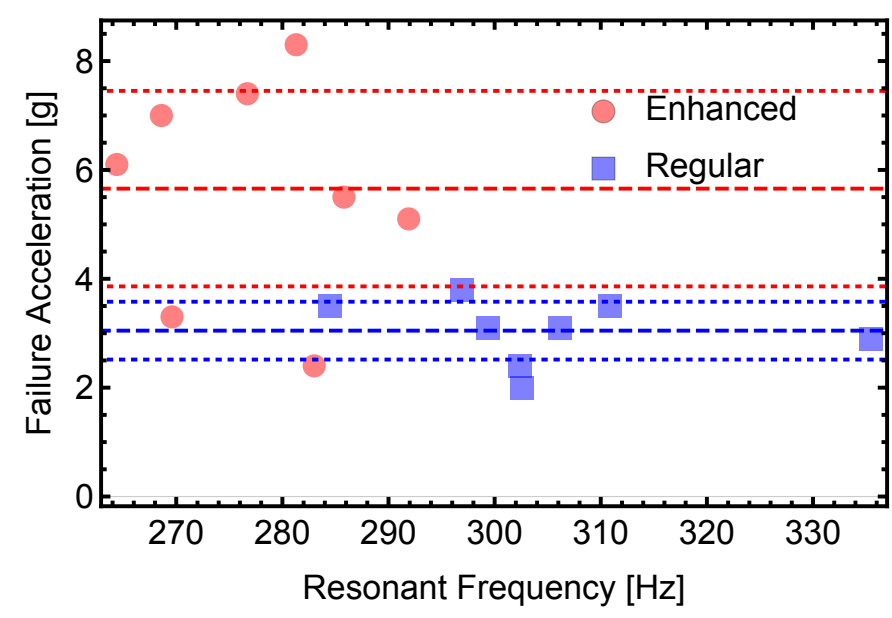

Fig. 12. Failure base acceleration for the enhanced and regular devices as function of beam resonant frequency. The dashed lines are the calculated mean value of the Weibull distributions and the dotted lines represent the corresponding standard deviation. The corner rounding process increases the failure acceleration by almost a factor of two.

radius of curvature to values around $1 \mu \mathrm{m}$. Similar results were reported by [31] where the burst pressure was increased from around 3 bar to 8 bar when the radius of curvature of the corner was etched to obtain a value of $5 \mu \mathrm{m}$. However, controlling the corner rounding by wet isotropic etching is difficult [30] and the use of plasma etching reduces the throughput due to the limited number of wafers that can be processed at the same time. These problems are overcome by the oxidation process used here as the radius of curvature can be predicted and the corner rounding can be obtained in a batch process.

\subsection{Electrical domain}

Impedance measurements in both the linear regime (without magnets), and the non-linear regime (with magnets implemented into the system) was carried out.

The capacitance of the piezoelectic layer was found before the ferromagnetic foil was mounted on the beam using an impedance measurement in the frequency range from $20 \mathrm{~Hz}$ to $1100 \mathrm{~Hz}$ and a value of $C=4.5 \mathrm{nF}$ was determined. The relative permittivity of the piezoelectric material was calculated to $\epsilon_{r}=10.05$, which is in accordance with the value found by Elfrink et al. [6].

For the linear case, a frequency sweep around the resonant frequency of the beam was performed, as shown in Fig 13, where the impedance magnitude and the phase are plotted on two different y-axes. The results show a resonant frequency, $f_{r}=240.6 \mathrm{~Hz}$ and the spring constant was calculated to $k_{\mathrm{eff}}=$ $98.7 \mathrm{~N} / \mathrm{m}$ using an effective mass of $m_{\mathrm{eff}}=43 \mathrm{mg}$.

The non-linear response was characterized by using the setup described in Section 2.2.2. Figures 14 and 15 show the impedance measurements obtained for a horizontal distance between the magnets and the beam of $a=1.15 \mu \mathrm{m}$ and $a=500 \mu \mathrm{m}$, respectively.

In the first case, Fig. 14, the magnetoelastic effect leads to softening of the beam, and the resonant frequency is

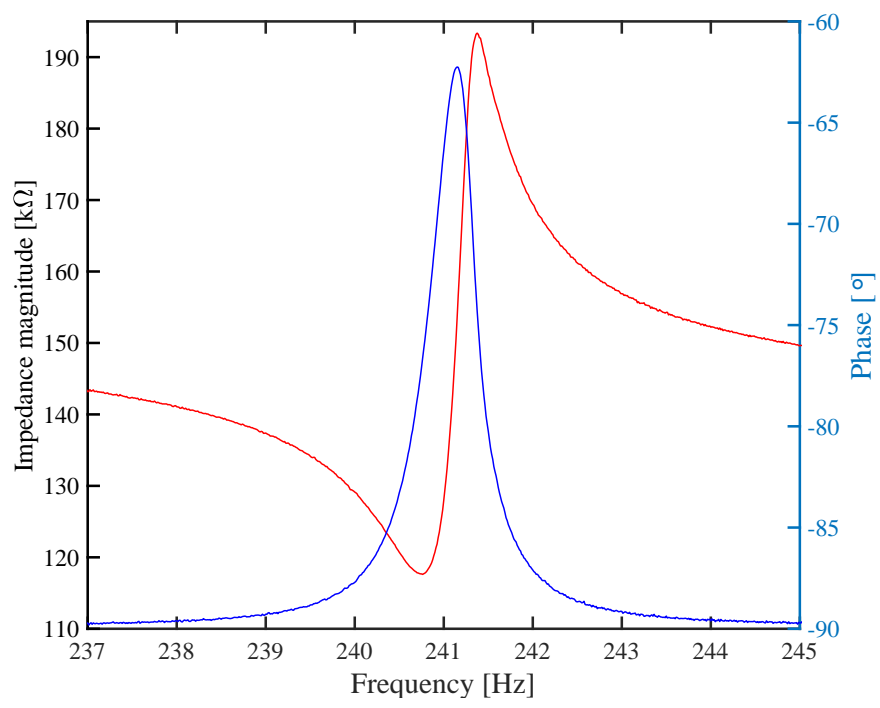

Fig. 13. Impedance measurement of the beam in the linear regime, i.e. with no magnetic force interacting with the beam.

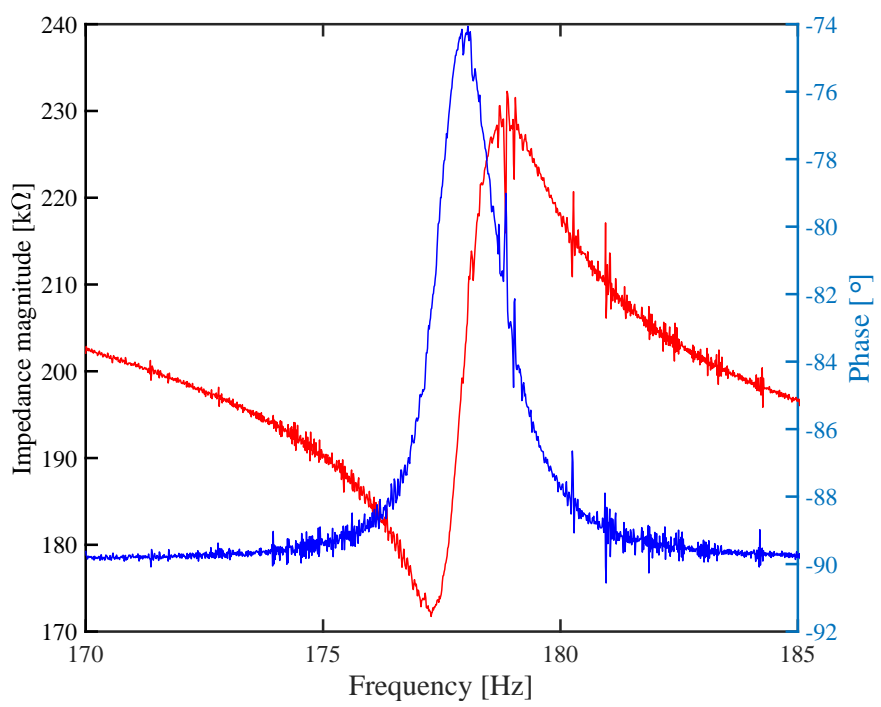

Fig. 14. Impedance measurement around the lowest non-linear resonant frequency due to the softening effect. The magnet-beam distance $a$ is $1.1 \mathrm{~mm}$ and the distance between the magnets is $b=520 \mu \mathrm{m}$.

$f_{r}=177.3 \mathrm{~Hz}$ and the effective spring constant is $k_{\text {eff }}=$ $53.58 \mathrm{~N} / \mathrm{m}$, which is 0.54 times the effective spring constant measured in the linear case. As can be seen in Fig. 14, there is some noise in the impedance spectrum compared to Fig. 13 and Fig. 15. The reason is that the larger the softening effect is, the more sensitive the beam becomes to any noise in the surroundings.

In the second case, Fig. 15, a resonant frequency of $f_{r}=$ $577.4 \mathrm{~Hz}$ is found, with an associated effective spring constant of $k_{\text {eff }}=568.3 \mathrm{~N} / \mathrm{m}$, which is 5.76 times larger the value found in the linear case showing a significant spring hardening.

Impedance spectra were measured for different distances between the magnets and beam, i.e. for different values of $a$, ranging from $a=6 \mathrm{~mm}$ to $a=400 \mu \mathrm{m}$. The distance 


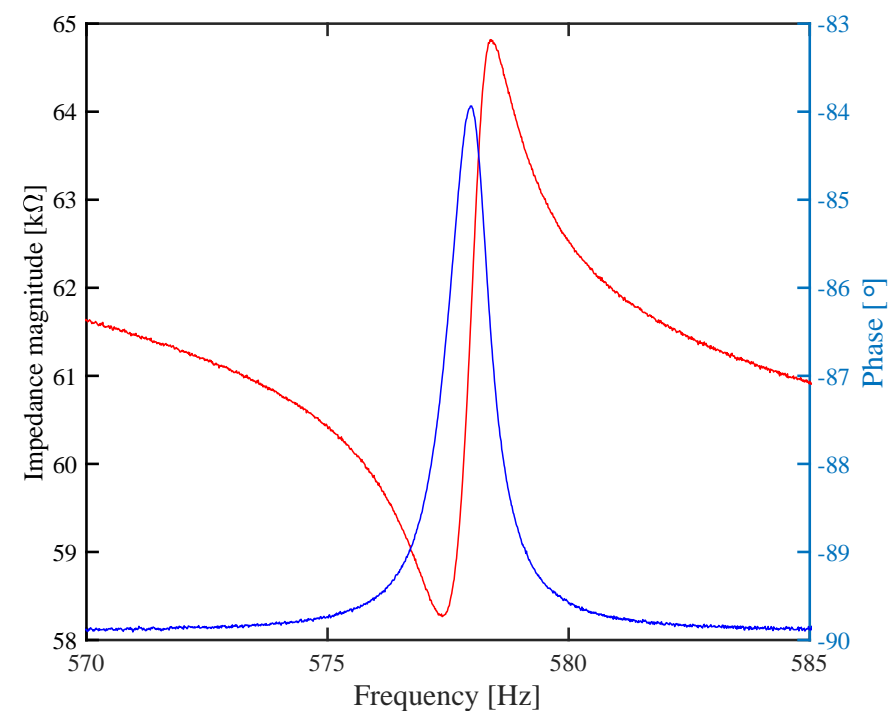

Fig. 15. Impedance measurement around the highest non-linear resonant frequency due to the hardening effect. The magnet-beam distance $a$ is $400 \mu \mathrm{m}$ and the distance between the magnets is $b=520 \mu \mathrm{m}$.

between the magnets was $b=520 \mu \mathrm{m}$. The results are shown in Fig. 16, where the resonant frequency of the beam, the impedance magnitude associated to the resonant frequency and the maximum phase are plotted as function of $a$. It can be seen that for large values of $a$ the associated values correspond to the linear regime, i.e. the magnetic force is not strong enough at that distance to influence the dynamics of the beam. At a distance of about $a=3 \mathrm{~mm}$ a small shift in the measured values begins. However, it is not until a value of about $a=2 \mathrm{~mm}$ is reached that a considerable effect takes place. Down until $a=1.15 \mathrm{~mm}$ a softening effect takes place and the resonant frequency consistently decreases until $f_{r}=177.3 \mathrm{~Hz}$ and the impedance magnitude increases as expected. For lower values of $a$ the resonant frequency increases and the impedance magnitude is lowered, which demonstrates a hardening effect in that region.

These results demonstrate that it is possible to obtain a significant spring softening in a miniature system. The needed distances between the beam and the magnets can be easily obtained in practice. The spring softening obtained will widen the frequency bandwidth where energy harvesting can be performed.

\section{CONCLUSION}

In conclusion, a cantilever-based piezoelectric magnetoelastic vibrational energy harvester with increased mechanical robustness has been presented. The energy harvester was fabricated using $\mathrm{KOH}$ etching of silicon to define the cantilever and ferromagnetic iron foils were used both as a proof mass and as part of the magnetoelastic design. A two-step lithographyfree fabrication process capable of increasing the mechanical robustness of the cantilever was developed. The increased mechanical robustness was obtained by rounding the sharp corner at the anchoring point of the beam, developed during

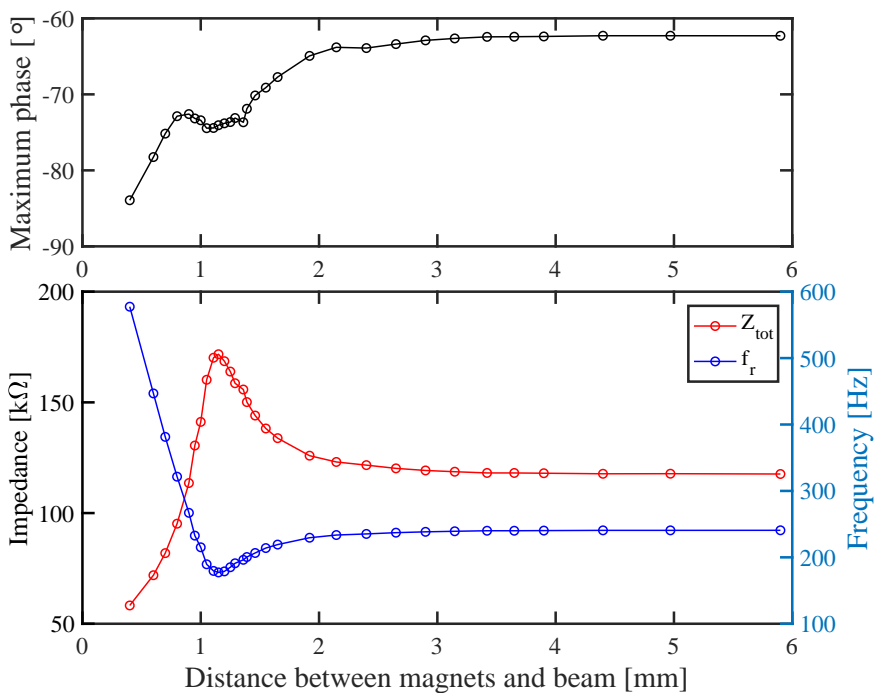

Fig. 16. Plot of the resonant frequency, impedance magnitude at the resonance frequency and maximum phase for a range of $a$ values. The distance between the magnets was $b=520 \mu \mathrm{m}$.

the $\mathrm{KOH}$ etch, by oxidation. A $1100 \mathrm{~nm}$ thick wet thermal oxide was grown once the beam had been defined, and the two dimensional diffusion of the oxidant led to a corner rounding increasing the radius of curvature to $1.4 \mu \mathrm{m}$. The thermal process was simulated with the Silvaco ATHENA process simulation software and the simulation could accurately predict the corner rounding as demonstrated by comparison of the simulated structure with a SEM image of the fabricated device. The radius of curvature of the corner depends linearly on the thickness of the oxide and as a rule of thumb the radius of curvature is equal to the thickness of the oxide. The corner rounding increased the mechanical robustness of the beam and the enhanced beams withstood a base mean acceleration of $5.7 \mathrm{~g}$ at their resonant frequency, which is almost twice as much as what was observed for the devices without corner rounding where the mean failure base acceleration was $3.0 \mathrm{~g}$.

The devices were also characterized electrically by impedance measurements, which demonstrated the magnetoelastic behaviour of the beam and both spring softening and hardening was observed depending on the distance between the magnets used and the tip of the beam. The lowest resonant frequency achieved was $177 \mathrm{~Hz}$, which occurred at a distance between the tip of the beam and the magnets of $a=1.15 \mathrm{~mm}$, a distance that is easily obtained during assembly of the energy harvesting system.

\section{REFERENCES}

[1] Y. Tada, "Experimental characteristics of electret generator, using polymer film electrets," Japanese Journal of Applied Physics, vol. 31, no. 3R, p. 846, 1992.

[2] S. Roundy, P. K. Wright, and J. Rabaey, "A study of low level vibrations as a power source for wireless sensor nodes," Computer Communications, vol. 26, no. 11, pp. 1131-1144, 2003.

[3] S. Boisseau, G. Despesse, T. Ricart, E. Defay, and A. Sylvestre, "Cantilever-based electret energy harvesters," Smart Materials and Structures, vol. 20, no. 10, p. 105013, 2011 
[4] M. El-Hami, P. Glynne-Jones, N. White, M. Hill, S. Beeby, E. James, A. Brown, and J. Ross, "Design and fabrication of a new vibration-based electromechanical power generator,' Sensors and Actuators A: Physical, vol. 92, no. 1, pp. 335-342, 2001.

[5] S. P. Beeby, R. Torah, M. Tudor, P. Glynne-Jones, T. O'Donnell, C. Saha, and S. Roy, "A micro electromagnetic generator for vibration energy harvesting," Journal of Micromechanics and Microengineering, vol. 17, no. 7, p. $1257,2007$.

[6] R. Elfrink, T. Kamel, M. Goedbloed, S. Matova, D. Hohlfeld, R. Van Schaijk, and R. Vullers, "Vibration energy harvesting with aluminum nitride-based piezoelectric devices," in Proceedings of the PowerMEMS Workshop, Sendai, 2008, pp. 249-52.

[7] A. Erturk and D. J. Inman, "An experimentally validated bimorph cantilever model for piezoelectric energy harvesting from base excitations," Smart Materials and Structures, vol. 18, no. 2, p. 025009, 2009.

[8] S. Priya, "Advances in energy harvesting using low profile piezoelectric transducers," Journal of Electroceramics, vol. 19, no. 1, pp. 167-184, 2007.

[9] E. Iborra, J. Olivares, M. Clement, L. Vergara, A. Sanz-Hervás, and J. Sangrador, "Piezoelectric properties and residual stress of sputtered AlN thin films for MEMS applications," Sensors and Actuators A: Physical, vol. 115, no. 2, pp. 501-507, 2004.

[10] P. Wang, H. Du, S. Shen, M. Zhang, and B. Liu, "Deposition, characterization and optimization of zinc oxide thin film for piezoelectric cantilevers," Applied Surface Science, vol. 258, no. 24, pp. 9510-9517, 2012.

[11] S. Roundy, E. S. Leland, J. Baker, E. Carleton, E. Reilly, E. Lai, B. Otis, J. M. Rabaey, P. K. Wright, and V. Sundararajan, "Improving power output for vibration-based energy scavengers," IEEE Pervasive computing, vol. 4, no. 1, pp. 28-36, 2005.

[12] P.-h. Wang, S.-w. Shi, and H.-j. Du, "Fabrication and performance of $\mathrm{ZnO}$ piezoelectric cantilever for vibration energy harvesting," in Piezoelectricity, Acoustic Waves, and Device Applications (SPAWDA), 2015 Symposium on. IEEE, 2015, pp. 147-151.

[13] S.-C. Lin and W.-J. Wu, "Fabrication of PZT MEMS energy harvester based on silicon and stainless-steel substrates utilizing an aerosol deposition method," Journal of Micromechanics and Microengineering, vol. 23, no. 12, p. 125028, 2013

[14] S. Roundy, P. K. Wright, and J. Rabaey, "A study of low level vibrations as a power source for wireless sensor nodes," Computer Communications, vol. 26, no. 11, pp. 1131-1144, 2003.

[15] J. Gere and S. Timoshenko, Mechanics of materials, ser. General Engineering Series. PWS Pub Co., 1997.

[16] R. Budynas, W. Young, and A. Sadegh, Roark's Formulas for Stress and Strain, 8th Edition. McGraw-Hill Education, 2011.

[17] W. D. Pilkey and D. F. Pilkey, "Peterson's stress concentration factors, third edition," Peterson's Stress Concentration Factors, Third Edition, pp. 1-522, 2008.

[18] M. T. Todaro, F. Guido, V. Mastronardi, D. Desmaele, G. Epifani, L. Algieri, and M. De Vittorio, "Piezoelectric MEMS vibrational energy harvesters: Advances and outlook," Microelectronic Engineering, vol. 183-184, pp. 23-26, 2017.

[19] F. Moon and P. J. Holmes, "A magnetoelastic strange attractor," Journal of Sound and Vibration, vol. 65, no. 2, pp. 275-296, 1979.

[20] M. Ferrari, V. Ferrari, M. Guizzetti, B. Andò, S. Baglio, and C. Trigona, "Improved energy harvesting from wideband vibrations by nonlinear piezoelectric converters," Sensors and Actuators A: Physical, vol. 162, no. 2, pp. 425-431, 2010.

[21] A. Lei and E. V. Thomsen, "Wideband piezomagnetoelastic vibration energy harvesting," in Journal of Physics: Conference Series, vol. 557, no. 1. IOP Publishing, 2014, p. 012121.

[22] J. I. Tam and P. Holmes, "Revisiting a magneto-elastic strange attractor," Journal of Sound and Vibration, vol. 333, no. 6, pp. 1767-1780, 2014

[23] D. S. Nguyen and E. Halvorsen, "Analysis of vibration energy harvesters utilizing a variety of nonlinear springs," Proceedings of the PowerMEMS, vol. 10, pp. 331-334, 2010.

[24] R. Ramlan, "Effects of non-linear stiffness on performance of an energy harvesting device," Ph.D. dissertation, University of Southampton, 2009.

[25] M. S. Soliman, E. M. Abdel-Rahman, E. F. El-Saadany, and R. R. Mansour, "A design procedure for wideband micropower generators," Journal of Microelectromechanical Systems, vol. 18, no. 6, pp. 12881299, 2009.

[26] K. Morimoto, I. Kanno, K. Wasa, and H. Kotera, "High-efficiency piezoelectric energy harvesters of c-axis-oriented epitaxial PZT films transferred onto stainless steel cantilevers," Sensors and Actuators A: Physical, vol. 163, no. 1, pp. 428-432, 2010.

[27] S.-C. Lin and W.-J. Wu, "Piezoelectric micro energy harvesters based on stainless-steel substrates," Smart Materials and Structures, vol. 22 , no. 4, p. 045016, 2013.

[28] M. A. Meitl, X. Feng, J. Dong, E. Menard, P. M. Ferreira, Y. Huang, and J. A. Rogers, "Stress focusing for controlled fracture in microelectromechanical systems," Applied Physics Letters, vol. 90, no. 8, p. 083110, 2007.

[29] W. Chan, K. Sooriakumar, K. Bartholomew, T. S. Savage, and T. Vanhoy, "An investigation of corner rounding to strengthen silicon micromachined pressure sensor for fusion bonded wafers," Proceedings of the Third International Symposium on Semiconductor Wafer Bonding: Physics and Applications, pp. 528-34, 528-534, 1995.

[30] K. Sooriakumar, W. Chan, T. S. Savage, and C. Fugate, "A comparative study of wet versus dry isotropic etch to strengthen silicon micromachined pressure sensor," Proceedings of the Second International Symposium on Microstructures and Microfabricated Systems, pp. 25965, 259-265, 1995.

[31] H. D. Ngo, A. T. Tham, M. Simon, and E. Obermeier, "Corner rounding to strengthen silicon pressure sensors using DRIE," in 2008 IEEE Sensors, Oct 2008, pp. 1576-1579.

[32] W. Weibull, "A statistical distribution function of wide applicability," Journal of Applied Mechanics, vol. 18, no. 3, pp. 293-297, 1951.

[33] DinoCapture Users Manual, AnMo Electronics Corporation, Inc., 17F, No. 97, Sec. 4, ChongHsin Rd., Sanchong Dist., New Taipei City, 241 Taiwan, 2017. [Online]. Available: http://www.dino-lite.com

[34] A. Erturk and D. J. Inman, Piezoelectric energy harvesting. John Wiley \& Sons, 2011, pg. 27.

[35] ATHENA Users Manual, SILVACO, Inc., 4701 Patrick Henry Drive, Bldg. 2 Santa Clara, CA 95054, 2010. [Online]. Available: http://www.silvaco.com

\section{APPENDIX}

This appendix contains the simulation code for a typical corner rounding study.

\# Corner rounding simulation

go athena

\# Define the grid in the y-direction

\# (vertical, in units of um)

line $y$ loc $=0 \quad$ spac $=0.1$

line $y$ loc $=10 \quad$ spac $=0.1$

line $y$ loc $=20 \quad \mathrm{spac}=2$

\# Define the grid in the $\mathrm{x}$-direction

\# (horisontal, in units of um)

line $x$ loc $=-10 \quad \mathrm{spac}=.5$

line $x$ loc $=-5 \quad$ spac $=0.1$

line $x$ loc $=5 \quad$ spac $=0.1$

line $x$ loc $=10 \quad$ spac $=0.5$

\#

\# Define the wafer

\# n-type silicon, highly doped

\#

init silicon phosphor I

resistivity $=0.0015$ orientation $=100$

\# $\mathrm{KOH}$ etch

etch silicon start $x=-3 \quad y=0.00$

etch cont $\mathrm{x}=3 \mathrm{y}=8.485$

etch done $\mathrm{x}=10.00 \mathrm{y}=8.485$ 


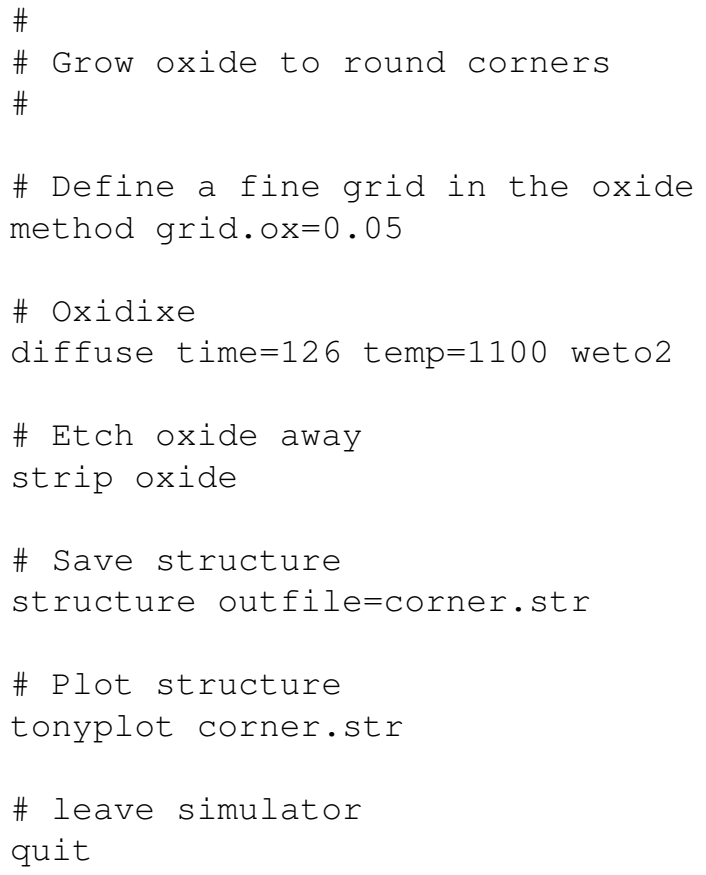

